\title{
Photopolymerization of Diacetylene Lipid Bilayers and Its Application to the Construction of Micropatterned Biomimetic Membranes
}

\author{
Kenichi Morigaki,,,$+\dagger$ Tobias Baumgart,, UIrich J onas, \\ Andreas Offenhäusser, $§$ and Wolfgang Knoll \\ Max-Planck-Institut für Polymerforschung, Ackermannweg 10, 55128 Mainz, Germany
}

Received December 18, 2001. In Final Form: March 6, 2002

\begin{abstract}
Photopolymerization of diacetylene-containing amphiphiles in substrate-supported bilayers has been studied in connection with the development of a new fabrication strategy of micropatterned biomimetic membranesystems. Twotypes of amphiphilic diacetylenemolecules were compared, one being a monoalkyl phosphate, phosphoric acid monohexacosa-10,12-diynyl ester (1), and the other being a phospholipid, 1,2-bis(10,12-tricosadiynoyl)-sn-glycero-3-phosphocholine (2). The bilayers of monomeric diacetylene amphiphiles were deposited onto substrates by the Langmuir-Blodgett/Langmuir-Schaefer methods. Both amphiphiles could be polymerized successfully on oxide and polymer-coated substrates. However, the phospholipid (2) showed a markedly higher mechanical stability of the polymerized bilayer films compared with themonoal kyl phosphate(1). Micropatterns wereimposed in the bilayers by using a physical mask to protect the monomeric lipids partially upon UV irradiation. In the case of $\mathbf{2}$, monomeric bilayers could beremoved selectively by ethanol after thelithographic photopolymerization, resulting in theformation of wells between the polymerized bilayers. These wells were filled with fluid phosphatidylcholine bilayers by fusion of vesicles. The rigid polymeric bilayer and the fluid biomimetic bilayer could be integrated as a compositebilayer membranewith defined spatial patterns, offering new possi bilities to construct complex and versatile biomimetic membrane systems.
\end{abstract}

\section{Introduction}

Substrate-supported planar lipid bilayers (abbreviated as SPBs in the following) represent a unique type of biomimetic membranesystem, wherea singlelipid bilayer is attached toa solid surfaceeither by physical interactions or by chemical bonds. SPBs are generally designed such that the bilayer membranemi mics somekey physical and chemical properties of the biological membrane(e.g., lipid composition, fluidity, resistance to nonspecific protein adsorption, etc.). ${ }^{1-4}$ Such artificial membrane systems provide attractive tools both for biophysical studies and for biomedical/environmental applications. ${ }^{2,3}$ For example, attempts have been madetointegratemembrane proteins in their active form in order to monitor their activities and develop sensor/diagnostic applications based on specific ligand/receptor bindings. ${ }^{5}$

Compared with other formats of artificial membrane systems (lipid vesicles, black lipid membranes, etc.), SPBs possess the unique feature that they can potentially be created in micropatterned geometries, in anal ogy to the integrated circuit on a silicon wafer. The patterning of SPBs has been reported by using several approaches including the modification of solid substrates and the

† Current address: National Institute of Advanced Industrial Science and Technology, I keda 563-8577, J apan. Fax: +81-72751-9628. E-mail: morigaki-kenichi@aist.go.jp.

₹ Current address: School of Applied and Engineering Physics, Cornell University, Clark Hall, Ithaca, NY 14853.

$\S$ Current address: Institute for Thin Films \& Interfaces, Forschungszentrum J ülich, 52425 J ülich, Germany.

(1) Chapman, D. Langmuir 1993, 9, 39-45.

(2) Sackmann, E. Science (Washington, D.C.) 1996, 271, 43-48.

(3) Plant, A. Langmuir 1999, 15, 5128-5135.

(4) Wisniewski, N.; Reichert, M. Colloids Surf., B 2000, 18, 197219.

(5) Heyse, S.; Ernst, O. P.; Dienes, Z.; Hofmann, K. P.; Vogel, H. Biochemistry 1998, 37, 507-522. microcontact printing technique. ${ }^{6-11}$ We have recently reported a novel method for the mi cropatterning of SPBs based on the lithographic photopolymerization of a diacetylene phospholipid within bilayers. ${ }^{12}$ Photopolymerization cross-linked li pid mol ecules selectively where UV light was irradiated, and the nonirradiated bilayer membranes, which consisted of monomers, could be removed by an organic solvent or by a detergent solution. The resulting wells between polymeric bilayers could be filled with phospholipid bilayers that possess lateral fluidity. The polymeric bilayers acted as a template to incorporate new biomimetic lipid bilayer membranes in a spatially defined manner.

Polymerization of lipidic molecules in Iyotropic, selfassembled aggregations (lamellar, cubic, inverted hexagonal, etc.) has attracted considerable attention in the last several decades. ${ }^{13-15}$ In particular, stabilization of lipid vesicles (liposomes) by polymerization of lipids has been studied extensively in connection with the potential application for encapsulation of medicinal materials (drug

(6) Groves, J . T.; UIman, N.; Boxer, S. G. Science(Washington, D.C.) 1997, 275, 651-653.

(7) Wiegand, G.; J aworek, T.; Wegner, G.; Sackmann, E. Langmuir 1997, 13, 3563-3569.

(8) J enkins, A. T. A.; Boden, N.; Bushby, R.J .; Evans, S. D.; Knowles, P. F.; Miles, R. E.; Ogier, S. D.; Schönherr, H.; Vancso, G. J . J . Am. Chem. Soc. 1999, 121, 5274-5280.

(9) Hovis, J. S.; Boxer, S. G. Langmuir 2000, 16, 894-897. 8131.

(10) Cremer, P. S.; Yang, T. J . Am. Chem. Soc. 1999, 121, 8130-

(11) Groves, J . T.; Mahal, L. K.; Bertozzi, C. R. Langmuir 2001, 17, $5129-5133$.

(12) Morigaki, K.; Baumgart, T.; Offenhäusser, A.; Knoll, W. Angew. Chem., Int. Ed. Engl. 2001, 40, 172-174.

(13) Ringsdorf, H.; Schlarb, B.; Venzmer, J . Angew. Chem., Int. Ed. Engl. 1988, 27, 113-158.

(14) Singh, A.; Schnur, J . M. In Phospholipids Handbook; Cevc, G., Ed.; Marcel Dekker: New York, 1993.

(15) O'Brien, D. F.; Armitage, B.; Benedicto, A.; Bennett, D. E.; Lamparski, H. G.; Lee, Y. S.; Srisiri, W.; Sisson, T. M. Acc. Chem. Res. 1998, 31, 861-868. 


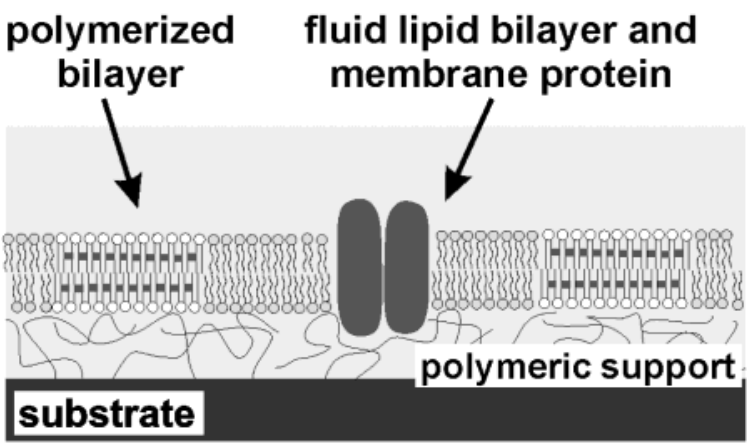

Figure 1. Schematic illustration of a substrate-supported biomimeticmembranethat consists of patterned polymericlipid bilayers and fluid li pid bilayers supported by a polymeric spacer layer.

delivery). ${ }^{16}$ Lipid molecul es having various moieties (e.g., dienoyl, sorbyl, diacetylene, etc.) were synthesized and polymerized in situ within the bilayers. U pon polymerization, the bilayers became significantly less soluble in organic solvents or detergent solutions. ${ }^{17-20}$ In addition, decrease in the lateral diffusion constant and the permeation coefficient of the bilayers was observed. ${ }^{21,22}$

Micropatterning of SPBs by lithographic photopolymerization of lipid bilayers aims to achieve both spatial control and stabilization of biomimetic membranes. One distinctive feature of this approach in comparison with previously reported micropatterning methods is the fact that the pattern is imprinted in the bilayer membrane. It enables the patterned two-dimensional structure to be separated from the substrate by a thin polymeric layer, which is often necessary for the successful incorporation of membrane proteins into SPBs. ${ }^{23-27}$ An envisioned membrane structure is depicted in Figure 1 . I ntegrated membrane systems with defined spatial patterns should enable the reproduction of various complex biological signal transduction events in an artificial platform. Nevertheless, there are various technical challenges to be addressed to create such complex structures in a controlled way.

Herein we report on the photopolymerization process of diacetylene lipid bilayers on solid surfaces. We have compared the photopolymerization behaviors of two diacetylene-containing amphiphiles, a monoalkyl phosphate (1) and a phosphol ipid (2) (Scheme1) on oxideand polymercoated substrates. The choice of the diacetyl ene group for studying polymerization of bilayers was based on the following two properties. (i) Poly(diacetylenes) form long conjugation of ene-yne backbones that absorbUV/visible light strongly. F urthermore, certain types of poly(diacety-

(16) Freeman, F. J .; Chapman, D. In Liposomes as drug carriers; Gregoriadis, G., Ed.; J ohn Wiley \& Sons: New Y ork, 1988; pp 821-839.

(17) J ohnston, D. S.; McLean, L. R.; Whittam, M. A.; Clark, A. D.; Chapman, D. Biochemistry 1983, 22, 3194-3202.

(18) Leaver, J .; Alonso, A.; Durrani, A. A.; Chapman, D. Biochim. Biophys. Acta 1983, 732, 210-218.

(19) Takeoka, S.; Ohgushi, T.; Tsuchida, E. Macromolecules 1995, $28,7660-7666$.

(20) Sisson, T. M.; Lamparski, H. G.; Kölchens, S.; Elayadi, A.; O'Brien, D. F. Macromolecules 1996, 29, 8321-8329.

(21) Sackmann, E.; Eggl, P.; Fahn, C.; Bader, H.; Ringsdorf, H. Schollmeier, M. Ber. Bunsen-Ges. Phys. Chem. 1985, 89, 1198-1208.

(22) Kölchens, S.; Lamparski, H.; O'Brien, D. F. Macromol ecules 1993 26, 398-400.

(23) Spinke, J .; Yang, J .; Wolf, H.; Liley, M.; Ringsdorf, H.; Knoll, W. Biophys. J . 1992, 63, 1667-1671.

(24) Wong, J . Y.; Majewski, J .; Seitz, M.; Park, C. K.; Israelachvili, J.: Smith, G. S. Biophys. J. 1999, 77, 1445-1457.

(25) Wong, J . Y.; Park, C. K.; Seitz, M.; I sraelachvili, J . Biophys. J 1999, 77, 1458-1468.

(26) Wagner, M. L.; Tamm, L. K. Biophys. J . 2000, 79, 1400-1414.

(27) Sackmann, E.; Tanaka, M. TIBTECH 2000, 18, 59-64.

\section{Scheme 1. Chemical Structures of Diacetylene-Containing Monoalkyl Phosphate (1) and Phospholipid (2) and Their Photopolymerization} Scheme
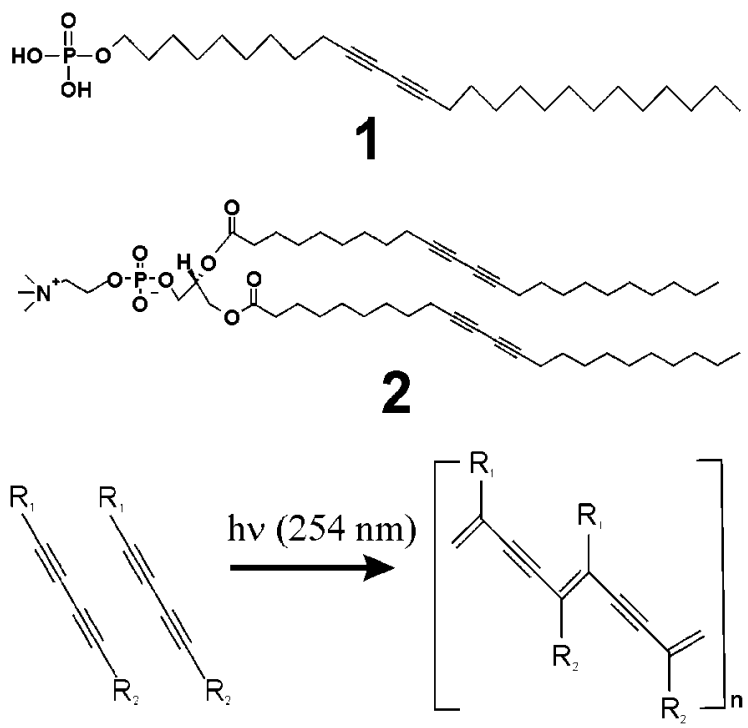

lenes) show a marked fluorescence. These properties facilitate the characterization of the polymers both spectroscopically and microscopically. (ii) Diacetylene mol ecules polymerize only in a highly ordered state (i.e., topochemical or solid-state polymerization), ${ }^{28,29}$ and the polymerization is very sensitive to the factors that affect the molecular packing. ${ }^{30-32}$ In the case of SPBs, the molecular packing within the bilayer is influenced by the substrate. ${ }^{33,34}$ Both $\mathbf{1}$ and $\mathbf{2}$ showed distinctive polymerization behaviors, depending on thetype of substrate. The comparison of twodifferent types of amphiphiles, onebeing a singlealkyl chain lipid and theother being a phopholipid with two alkyl chains, yiel ded valuable insight into some important aspects for the development of patterned biomimetic membranes based on the lithographic photopolymerization of bilayers. Thesingl echain amphiphile 1 showed a markedly higher reactivity compared with the phospholipid 2. The conjugation of the ene-yne backbones was also longer in polymerized $\mathbf{1}$ bilayers accordingly and showed distinctive spectral features (mixtures of the blue and red polymers) depending on the underlying substrates. Therefore, bilayers of $\mathbf{1}$ provided a convenient system to systematically study the influence of the substrate materials on the photopolymerization. The phospholipid $\mathbf{2}$, on the other hand, proved to be a suitable material for the purpose of the micropatterning of SPBs due to the high stability of polymerized bilayers. The comparison of stability between polymerized $\mathbf{1}$ and 2 suggested the importance of cross-links within the polymeric backbones (only possible with 2 ) for the stabilization of the bilayer membranes.

(28) Bloor, D.; Chance, R. R., Eds. Polydiacetylenes: Synthesis, Structure and Electronic Properties; Martinus Nijhoff Publishers: Dordrecht/Boston/Lancaster, 1985.

(29) Cantow, H.-J ., Ed. Polydiacetylenes; Springer-Verlag: Berlin, 1984

(30) Charych, D. H.; Nagy,J . O.; Spevak, W.; Bednarski, M. D. Science (Washington, D.C.) 1993, 261, 585-588.

(31) J onas, U.; Shah, K.; Norvez, S.; Charych, D. H. J . Am. Chem Soc. 1999, 121, 4580-4588.

(32) Okada, S.; Peng, S.; Spevak, W.; Charych, D. Acc. Chem Res. 1998, 31, 229-239.

(33) Kuriyama, K.; Kikuchi, H.; Kajiyama, T. Langmuir 1996, 12 2283-2288.

(34) Britt, D. W.; H ofmann, U. G.; Möbius, D.; Hell, S. W. Langmuir 2001, 17, 3757-3765. 


\section{Materials and Methods}

2.1. Materials. Diacetylene-containing monoalkyl phosphate, phosphoric acid monohexacosa-10,12-diynyl ester (1), was a gift from Professor Hel mut Ringsdorf. Diacetylenephospholipid, (1,2bis(10,12-tricosadiynoyl)-sn-glycero-3-phosphocholine) (2) and phosphatidylchol inefrom egg yolk (egg-PC) were purchased from Avanti Polar Lipids (Alabaster, AL). NBD-PE (N-(7-nitrobenz2-oxa-1,3-diazol-4-yl )-1,2-di hexadecanoyl-sn-glycero-3-phosphoethanolamine) was purchased from Molecular Probes (Eugene, OR). Chitosan ( $\left.M_{w} 600000\right)$, polyethylimine(PEI; $\left.M_{w} 2000\right)$, and sodium dodecyl sulfate (SDS) were from Fluka (Buchs, Switzerland). Other chemicals were purchased from Sigma (St. Louis, MO). All the commercially obtained chemicals were used without further purification. As the substrates of SPB, we have used either slide glass (Menzel, Braunschweig, Germany), polished quartz (Hellma, Müllheim, Germany), or thermally oxidized silicon (oxide layer thickness 154-163 nm, IMM, Mainz, Germany).

2.2. SubstrateCleaning and Modification with Polymers. The substrates were cleaned first with a commercial detergent solution ( $0.5 \%$ Hellmanex/water, Hellma, Mühlheim, Germany), rinsed with deionized water, treated in a warm concentrated sulfuric acid ( $70{ }^{\circ} \mathrm{C}$ for $15 \mathrm{~min}$ ), rinsed with deionized water extensively, and then dried in a vacuum oven at $110^{\circ} \mathrm{C}$. This protocol resulted in sufficiently clean and hydrophilic surfaces for the adsorption of polymers and lipid bilayer membranes. Chitosan and PEI wereadsorbed onto thesubstrates from dilute aqueous sol utions (ca. $1 \mathrm{w} / \mathrm{w} \%$ ). I $n$ the case of chitosan, a di lute acetic acid solution ( $1 \mathrm{v} / \mathrm{v} \%$ ) was used to dissolve the polymer in a slightly acidic solution. The nonadsorbed polymers were removed from the substrate surfaces by extensive rinsing with the acetic acid solution and deionized water. The thickness of the adsorbed polymer layer was measured by ellipsometry for the dry films to be ca. $1 \mathrm{~nm}$ for chitosan and ca. $6 \mathrm{~nm}$ for PEI .

2.3. Preparation of Supported Planar Bilayers. Bilayers of monomeric $\mathbf{1}$ and $\mathbf{2}$ were deposited onto solid substrates from the air/water interface by the Langmuir-Blodgett (LB) and Langmuir-Schaefer (LS) methods using a KSV5000 L angmuir trough (KSV Instruments, Helsinki, Finland). ${ }^{35}$ Molecules of $\mathbf{1}$ and $\mathbf{2}$ were spread from dichloromethane/methanol (9:1 v/v) and chloroform solutions, respectively. The lipids formed stable monolayers at the air/water interface up to a surface pressure of $40 \mathrm{mN} / \mathrm{m}$ at $20^{\circ} \mathrm{C}$. The monolayers weretransferred onto sol id substrates at $35 \mathrm{mN} / \mathrm{m}$ (fully condensed state). The first monolayer was deposited by dipping and withdrawing the substrate vertically (LB method). The second monolayer was deposited onto the hydrophobic surface of the first monolayer by pressing the substrate horizontally through the monolayer at the air/water interface and dropping it into the subphase (LS method). The transferred film area corresponded well to the theoretical values for both the LB and LS procedures, indicating that bilayers were built on both oxide and polymeric substrates used. The bilayer structure is also supported by atomic force microscopy measurements. The samples were collected from the trough and stored in deionized water (in the dark) for the photopolymerization.

2.4. Photopolymerization of Bilayers. As thelight source, we used either a small low-pressure mercury lamp (2 W, UVP, Pen-Ray, Upland, CA) or a cross-linker (40 W, UVP CL-1000, Upland, CA) that emit strong UV light at $254 \mathrm{~nm}$. 1 could be polymerized in water in the presence of oxygen. In the case of $\mathbf{2}$, oxygen had to be removed from the aqueous solution prior to photopolymerization, since presence of oxygen inhibited the polymerization, presumably by quenching diacetyleneradicals. ${ }^{36}$ The polymerization was conducted in a closed system that comprised a water reservoir, a pump, and a cell (ca. $4 \mathrm{~mL}$ vol ume). The water reservoir was depleted from oxygen by purging with

(35) Another potential approach for thegeneration of adsorbed planar bilayer membranes, the vesicle fusion process, failed for these lipids, due to the high melting temperatures of the bilayers. At ambient temperature the bilayers were in the solidlike state. Even at el evated temperatures theattempts for vesiclefusion failed, presumably because of the stiffness of bilayers.

(36) Day, D.; Ringsdorf, H. J . Polym. Sci., Polym. Lett. Ed. 1978, 16, 205-210. argon. Oxygen-free water was circulated continuously by the pump $(3.8 \mathrm{~mL} / \mathrm{min})$ through the cell where the polymerization of the bilayers was conducted. The cell had two walls in the opposite sides, one being the sample (SPB was inside the cell) and the other being a quartz window through which UV light was illuminated. The desired patterns were transferred to the SPB in the photopolymerization process by illuminating the sample through a mask (a quartz slide with a patterned gold coating), which was put over the SPB (separated by a thin water layer) inside the cell. After sufficient circulation of deaerated water (typically $15 \mathrm{~min}$ ), the pump was stopped and the photopolymerization was started. The distance between the UV light source and the SPB was 5 and $20 \mathrm{~cm}$ for the Pen-Ray lamp and the cross-linker, respectively.

2.5. Incorporation of Fluid Bilayers into the Wells between Polymerized Bilayers. To remove unreacted monomers, the samples were immersed either in a detergent sol ution (80 mM SDS) or in ethanol for $10 \mathrm{~min}$ and rinsed extensively with deionized water. F or theincorporation of new lipid bilayers into thelipid-free wells, the vesiclefusion method was applied. ${ }^{37}$ Vesicle suspensions of egg-PC containing 1 mol \% NBD-PE (1 $\mathrm{mM}$ in a $0.05 \mathrm{M}$ phosphatebuffer with $0.1 \mathrm{M} \mathrm{NaCl}(\mathrm{pH}$ 7.0)) were extruded through a filter with pores of diameter $50 \mathrm{~nm}$ (Nuclepore, Corning, Acton, MA) by using an extruder (LiposoFast, Avestin, Ottawa, Canada). A $100-200 \mu \mathrm{L}$ portion of the filtrate was placed ontothe patterned $\mathbf{2}$ bilayer samples and sandwiched with another slide glass using a thin cover glass as a spacer in order toavoid scratchingthe patterned SPB surfaces. Thesamples were rinsed with a $0.05 \mathrm{M}$ phosphate buffer solution $(\mathrm{pH} 7.0$, containing $0.1 \mathrm{M} \mathrm{NaCl}$ ) after $5 \mathrm{~min}$.

2.6. UV-visAbsorption and F luorescenceSpectroscopy. UV-vis absorption spectra were obtained by using a PerkinElmer $\lambda 9$ spectrophotometer (Perkin-E Imer Anatlytical I nstruments, Shelton, CT). Fluorescence spectra were obtained with a SPEX F 212 (J obin-Y von, Edison, NJ ) by using the front-face mode (the samplewas placed at an angle of $45^{\circ}$ from theexcitation light incidence and the fluorescence was measured at $22.5^{\circ}$ ). For the fluorescence measurements of SPB, the samples were dried by nitrogen before the measurements. ${ }^{38}$ This procedure did not affect the fluorescencespectra of the poly(diacetylene) films (the obtained spectra were identical with the in situ measurements).

2.7. Microscopy Observation. Differential interference contrast images were taken with an Axi oskop microscope (Carl Zeiss, Göttingen, Germany) equipped with epi-illumination (HBO 50 lamp) and a color CCTV camera WL-CL 700/G (Panasonic, Osaka, J apan). For fluorescencemicroscopy, an invertoscope (IX70, Olympus, Tokyo, J apan) equipped with a mercury lamp (HBO100 , Olympus) was used. The excitation and observation wavelength were 488 and $530 \mathrm{~nm}$, respectively. Fluorescence microscopy images were obtained with a light-enhancing camera (Extended ISIS: Photonic Sciences, Robertsbridge, UK) and a frame grabber card (AG-5: Scion, Frederick, MD) and were processed using softwares Scion Image (Scion, Frederick, MD) or I mage Pro Plus (Media Cybernetics, Leiden, Netherlands).

\section{Results and Discussion}

3.1. Absorption/Fluorescence Spectra of Polymerized 1 in Suspended Bilayers. In the current study we have exploited fluorescence excitation spectroscopy extensively in order to detect and characterize the in situ polymerization. Fluorescence spectroscopy turned out to be highly convenient for reflective substrates such as oxidized silicon, where direct measurement of absorption spectra is difficult due to the optical interference. We describe first the absorption and fluorescence spectra of polymerized $\mathbf{1}$ in suspended bilayers (vesicles) as a

(37) Brian, A. A.; McConnell, H. M. Proc. Natl. Acad. Sci. U.S.A. 1984, 81, 6159-6163.

(38) This protocol was employed in order to minimize the effect of contamination. Since we characterized the polymerization of single bilayers by fluorescencespectroscopy, signals from foreign fluorophores (contaminants from the lab environment) often overwhelmed that of the samples and made reproducible measurements difficult. Foreign contaminants could diffuse onto the sample surfaces especially easily when we worked with wet samples. 
(A)

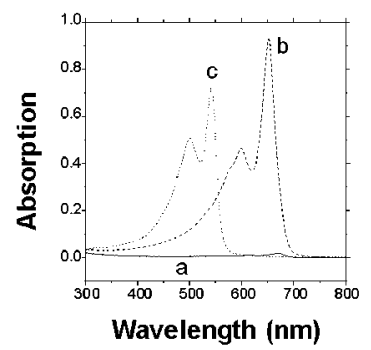

(C)

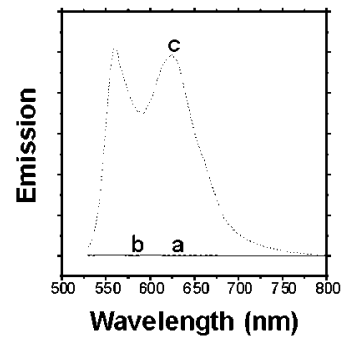

(B)

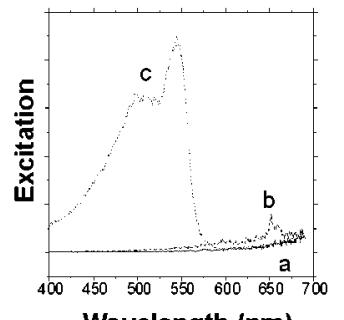

Wavelength (nm)

(D)

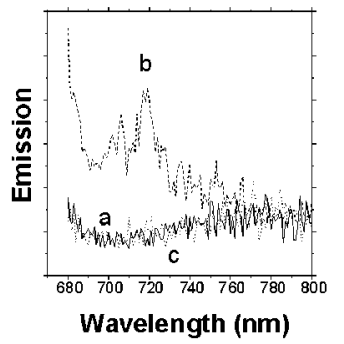

Figure 2. Absorption and fluorescence spectra of $\mathbf{1}$ vesicle suspensions (0.05 $\mathrm{mM}$ of $\mathbf{1}$ in water) were measured for (a) monomer, (b) blue polymer formed by UV irradiation (PenRay, 2 min), and (c) red polymer formed by heating the blue polymer: (A) absorption spectra $(0.05 \mathrm{mM}$, optical path length $=1 \mathrm{~cm}$ ); (B) fluorescence excitation spectra (emission was measured at $720 \mathrm{~nm}$ ); (C) fluorescence emission spectra (excitation at $500 \mathrm{~nm}$ ); (D) fluorescence emission spectra (excitation at $650 \mathrm{~nm}$ ).

referencefor further spectroscopicanalysis of SPB (Figure 2 ). Vesicle suspensi ons of $\mathbf{1}$ were prepared by dispersing the lipid molecules in water by probe sonication. Monomeric bilayers of $\mathbf{1}$ showed no absorption/fluorescence between 300 and $800 \mathrm{~nm}$ ((a) in F igure 2), and the sample solution was transparent. Thevesiclesuspensions became dark blue upon UV irradiation due to the formation of polymers that had strong absorption bands between 600 and $700 \mathrm{~nm}$ (blue polymer, (b) in Figure 2A). When the sample was heated, the polymeric bilayers underwent a structural transition and the absorption bands shifted toward shorter wavelengths (450-550 nm, (c) in Figure $2 \mathrm{~A})$. The color of the suspensi ons changed to pink or red accordingly (red polymer). This chromatic transition is known from literature as a consequence of the conformational relaxation of the polymer backbone and shortening of the effective conjugation length. ${ }^{39}$ Figure $2 \mathrm{~B}$ compares the fluorescence excitation spectra that were obtained for the same vesicle suspensions by measuring thefluorescenceemission intensity at 720 nmas a function of the excitation light wavelength. Whereas the red polymer had strong excitation bands between 425 and $575 \mathrm{~nm}$ (c) that roughly corresponded to its absorption bands, the blue polymer showed only a very weak excitation band between 600 and $700 \mathrm{~nm}$ (b). This observation also agrees with previous reports where the fluorescence was mainly observed from the red polymers. ${ }^{40-43}$ The fluorescence from the blue polymer is reduced, at least in part, due to increased radiationless

(39) Huo, Q.; Russell, K. C.; Leblanc, R. M. Langmuir 1999, 15, 39723980.

(40) Olmsted, J ., III; Strand, M. J . Phys. Chem. 1983, 87, 47904792.

(41) Warta, R.: Sixl, H. J . Chem. Phys. 1988, 88, 95-99.

(42) Carpick, R. W.; Mayer, T. M.; Sasaki, D. Y.; Burns, A. R. Langmuir 2000, 16, 4639-4647.

(43) Carpick, R. W.; Sasaki, D. Y.; Burns, A. R. Langmuir 2000, 16, $1270-1278$.

(A)

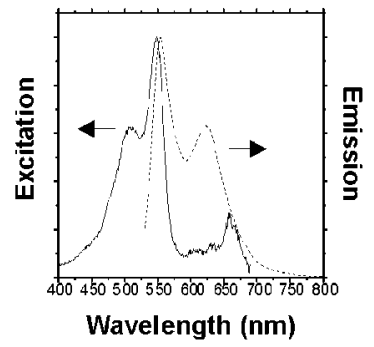

(B)

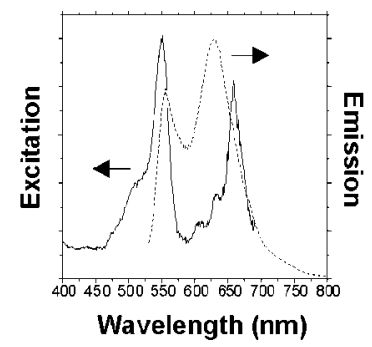

Figure 3. Fluorescence spectra of $\mathbf{1}$ bilayers polymerized on (A) quartz and (B) oxidized silicon substrates (UV irradiation, cross-linker, $400 \mathrm{~mJ}$ ). The excitation spectra (solid lines) were obtained by measuring the emission at $720 \mathrm{~nm}$. The emission spectra (dashed lines) were obtained by the excitation at 500 $\mathrm{nm}$.

deactivation processes. ${ }^{41}$ Emission spectra from the blue and red polymers have been al so measured (Figure 2C,D, excitation at $500 \mathrm{~nm}$ for (C) and $650 \mathrm{~nm}$ for (D), respectively). Fluorescence was indeed observed to arise from specific bands that corresponded to the red polymer ((c) in Figure2C) and thebluepolymer ((b) in Figure2D). ${ }^{44}$ The absorption and fluorescence spectra of suspended $\mathbf{1}$ bilayers are in good agreement with the reported spectroscopic observations.

3.2. Photopolymerization of 1 in SPB. Now weturn to the polymerization of the diacetylene amphiphiles in SPB adsorbed on various substrates. Figure 3 compares the fluorescence spectra of polymerized $\mathbf{1}$ on quartz and oxidized silicon substrates. Theobtained excitation spectra (solid lines in Figure 3) were considerably different from the vesicle samples (see Figure $2 \mathrm{~B}$ ) and showed strong dependency on thetype of substrates. The excitation band between 600 and $700 \mathrm{~nm}$ that should arise from the blue polymer was significantly enhanced in the case of oxidized silicon substrates (Figure 3B). As in the case of vesicles, the bluepolymer could betransformed into thered polymer by treatment with heat or organic solvents. Figure 4 shows the changes in absorption and fluorescence excitation spectra that were induced by immersing the polymeric bilayers in ethanol. The spectral bands between 600 and $700 \mathrm{~nm}$ disappeared after theethanol treatment, whereas the spectral bands at shorter wavelength retained the identical shape. ${ }^{45}$ It becomes clear from this result that theinitially formed polymeric bi layers contained both the red and blue forms. Upon treatment with ethanol they areconverted into a purely red stateduetosolvent-induced conformational changes in the polymer backbones. It is currently not clear how the two types of polymers coexist. They may be spatially resolved or molecularly mixed. It has been reported that red polymers are found at the rim of the blue polymer domains in Langmuir-Blodgett films. ${ }^{46}$ The distinctive spectral features on various substrates were reproducible and indicated different polymerization states. The different behaviors between quartz and oxidized silicon are rather surprising, since both materials should havesimilar chemical compositions. However, the difference persisted regardless of the surface cleaning methods for these substrates. Oxidized silicon substrates had slightly less hydrophilicsurfaces compared

(44) The fluorescence from the blue polymer was much weaker compared with that of the red polymer, as the low signal-to-noise ratio of line $b$ in Figure 2D indicates.

(45) The spectra were normalized, so that the spectral shapes could becompared. The bands for thered polymer after the ethanol treatment were generally stronger than those of the blue polymer.

(46) Bubeck, C.; Tieke, B.; Wegner, G. Ber. Bunsen-Ges. Phys. Chem. 1982, 86, 495-498. 
(A)

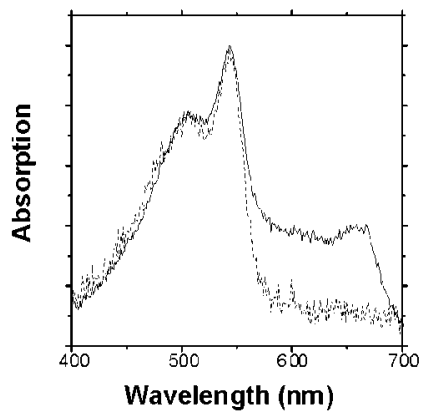

(B)

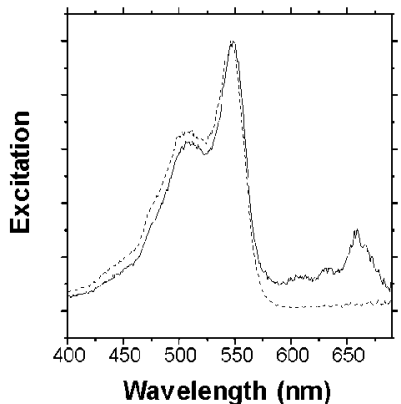

(C)

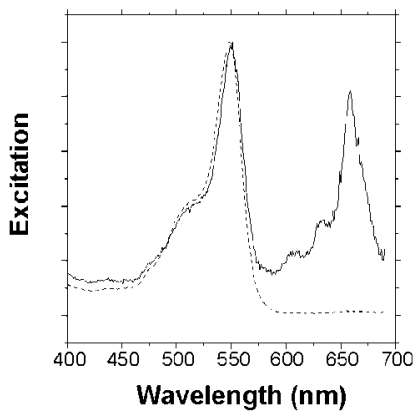

Figure 4. Spectral changes in polymeric 1 bilayers induced by ethanol treatment: $(A)$ absorption spectra of a single bilayer on a quartz substrate; (B) fluorescence excitation spectra (emission at $720 \mathrm{~nm}$ ) on a quartz substrate (the same sample as (A)); (C) fluorescence excitation spectra (emission at $720 \mathrm{~nm}$ ) on an oxidized silicon substrate; solid lines, UV polymerized bilayers before the ethanol treatment; dashed lines, after the ethanol treatment.

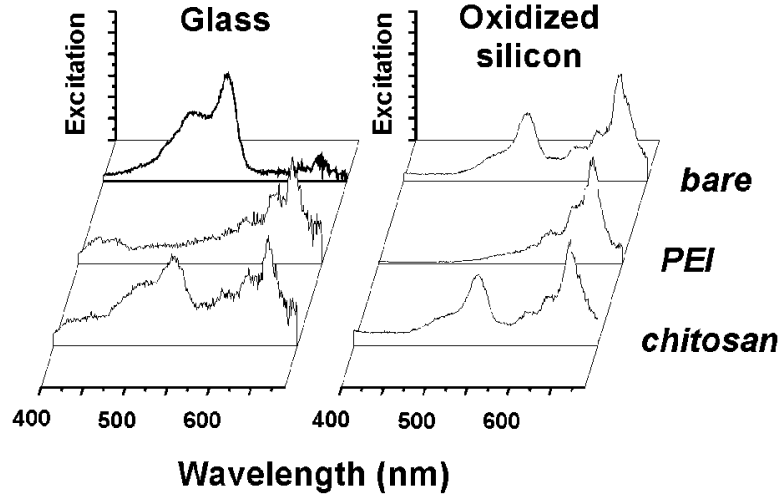

Figure 5. Fluorescence excitation spectra of polymerized $\mathbf{1}$ bilayers werecompared on glass and oxidized silicon substrates that weremodified with either PEI or chitosan (UV irradiation, cross-linker, $400 \mathrm{~mJ}$ ). The emission was measured at $720 \mathrm{~nm}$.

with quartz and glass substrates. ${ }^{47}$ This might have affected the molecular alignment of $\mathbf{1}$ bilayers. We observed for microscopesl ideglass substrates very similar polymerization behaviors as quartz substrates (seebelow).

Thesurface-dependent nature of the polymerization was moreevident when wemodified thesubstratesurface with thin polymer layers. As thesupporting polymericmaterial two water soluble polyel ectrolytes, PEI and chitosan, were used. Both polymers become positively charged by protonation at low $\mathrm{pH}(<6)$ and adsorb strongly onto negati vely charged substrates (glass and oxidized si licon) from the aqueous phase by el ectrostatic interactions. A thin polymeric layer remains on the substrate after extensive rinse with water. Bilayers of $\mathbf{1}$ were deposited ontothepolymericsupports by the LB/LS techniques, and they were subsequently polymerized by UV light. The obtained fluorescence excitation spectra are compared in Figure 5. The excitati on spectra of polymerized 1 on bare substrates showed distinctive spectral patterns on glass and oxidized silicon substrates. A strong excitation band between 600 and $700 \mathrm{~nm}$ was observed for the oxidized silicon substrate, as described above. The polymerized $\mathbf{1}$ on glass, on theother hand, showed strong excitation bands between 425 and $575 \mathrm{~nm}$, and those between 600 and 700 $\mathrm{nm}$ were relatively weak. ${ }^{48} \mathrm{~T}$ his spectral featureis similar to the polymerization on quartz (see Figure 3). When we used polyelectrolyte-modified glass or silicon as the

(47) Both quartz and oxidized silicon surfaces had contact angles that wereclosetozero. However, wenoted slightly different wettability as we observed the drying behavior of water droplets on the substrates.

(48) The fluorescence spectra of 1 bilayers on glass substrates had a larger noise because of the autofluorescence from glass that was subtracted from the sample spectra.
(A)

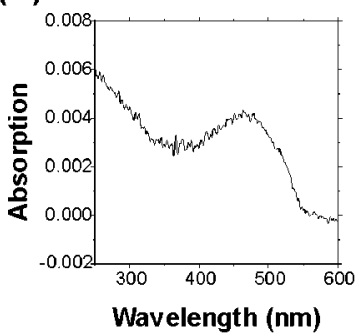

(B)

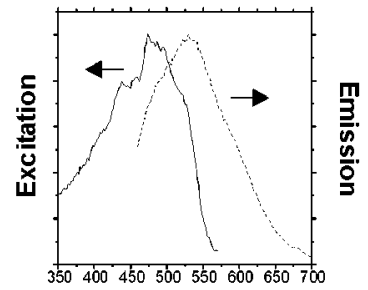

Wavelength $(\mathbf{n m})$

Figure 6. Absorption and fluorescence spectra of a polymeric 2 bilayer on a quartz substrate: (A) absorption spectrum (UV irradiation, Pen-Ray, $9 \mathrm{~min}$ ); (B) fluorescence excitation/ emission spectra (UV irradiation, Pen-Ray, $30 \mathrm{~min}$ ). The excitation spectrum (solid line) was obtained by measuring the emission at $600 \mathrm{~nm}$. The emission spectrum (dashed line) was obtained by the excitation at $430 \mathrm{~nm}$.

substrates, however, the excitation spectra were changed considerably. Polymerized $\mathbf{1}$ bilayers on PEI showed only the excitation bands between 600 and $700 \mathrm{~nm}$, indicating the formation of blue polymers. In constrast, chitosan substrates resulted in the formation of a mixture of the red polymers and the blue polymers (both spectral bands between 425 and $575 \mathrm{~nm}$ and between 600 and $700 \mathrm{~nm}$ were observed). These spectra were obtained regardless of the underlying substrate material (glass or oxidized silicon), indicating that the polymerization of $\mathbf{1}$ bilayers was determined by the type of the polymer (PEI or chitosan). Although the polymeric underlayers are very thin, they apparently determinethe surface properties of the substrates and direct the polymerization process of the bilayers. ${ }^{49}$

3.3. Photopolymerization of $\mathbf{2}$ in SPB. The bilayers of $\mathbf{2}$ were much less photoreactive compared with $\mathbf{1}$ bilayers. 2 polymerized only in an oxygen-freeatmosphere at much longer UV irradiation, whereas $\mathbf{1}$ polymerized rapidly in ambient atmosphere. The higher reactivity of $\mathbf{1}$ is presumably due to the ability of the single chain amphiphiles to pack in ordered crystalline bilayer structures. In the case of $\mathbf{2}$, the glycerol group of the phospholipid imposes sterical restrictions to the packing of the two alkyl chains bearing the diacetylene moieties..$^{50}$ The attenuated polymerization of $\mathbf{2}$ apparently resulted in a shorter conjugation length compared with $\mathbf{1}$ bilayers as seen in the absorption spectra (Figure 6A). When the

(49) These results also indicate that the reflectivity of silicon substrates did not cause the different polymerization behaviors of 1 bilayers on oxidized silicon substrates with respect to the bilayers on quartz/glass substrates.

(50) Lopez, E.; O'Brien, D. F.; Whitesides, T. H. J . Am. Chem. Soc. 1982, 104, 305-307. 


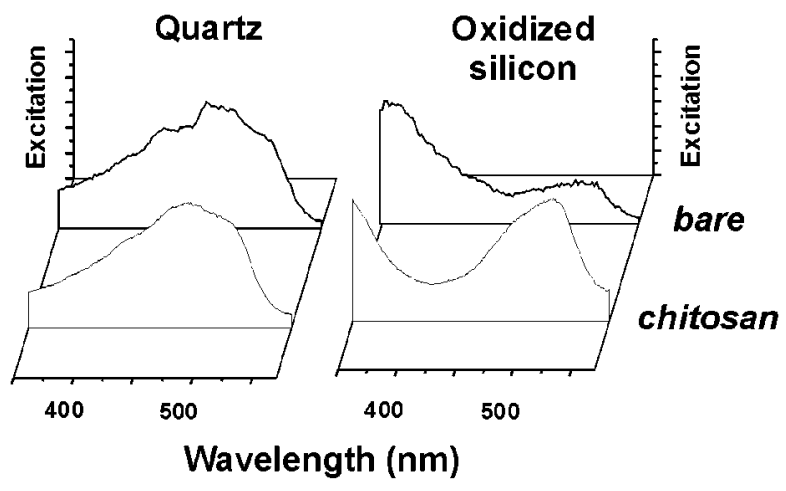

Figure 7. Fluorescence excitation spectra of polymerized 2 bilayers were compared on quartz and oxidized silicon substrates that were modified with a thin layer of chitosan (UV irradiation, Pen-Ray, 20 min). The emission was measured at $600 \mathrm{~nm}$.

bilayer was polymerized on quartz, the absorption maximum was observed around $470 \mathrm{~nm}$. The conjugated di acetylene pol ymer of $\mathbf{2}$ al so emitted strong fluorescence, as shown in Figure 6B. The shape of excitation spectrum corresponds roughly to the absorption spectrum. The polymerization on oxidized silicon resulted in an even shorter conjugation length (the excitation was observed predominantly below $400 \mathrm{~nm}$, see Figure 7).

Polymerization of $\mathbf{2}$ bilayers was also studied on a thin polymer layer (chitosan) that was adsorbed on quartz and oxidized silicon substrates. The fluorescence excitation spectra are shown in Figure 7. In the case of quartz substrates, the polymerization on the chitosan support occurred in the same way as the case of bare substrates. However, the presence of chitosan support had a more significant effect for oxidized silicon substrates. The excitation band between 450 and $550 \mathrm{~nm}$ was enhanced when the substrate was modified by the chitosan support. The bilayer on chitosan contained apparently a larger fraction of the polydiacetylene species with a longer conjugation length. These results show that the polymerization behavior of bilayers $\mathbf{2}$ is also strongly affected by the presence of a polymeric support layer.

3.4. Lithographic Photopolymerization of the Bilayers and Its Application to the Construction of Micropatterned Biomimetic Membranes. By protecting the monomeric bilayer selectively with a mask upon UV light exposure, we could create patterns of polymeric bilayer domains. A differential interference contrast microscope image of the patterned $\mathbf{1}$ bilayer is shown in Figure 8. The fine texture with the bright granules observed in the polymerized areas corresponds to crystalline domains of polymeric $\mathbf{1}$. This texture was also found in the fluorescence microscopy. ${ }^{51}$ The polymeric bilayers of $\mathbf{1}$ weremechanical ly unstabl eand peel ed off easily from the substrate when the samples were rinsed in water.

Figure 9A shows fluorescencemicrographs of patterned $\mathbf{2}$ bilayers. The polymerized areas are bright due to fluorescence from the conjugated polydiacetylene backbones. Polymerized bilayers of $\mathbf{2}$ appeared more homogeneous compared with those from $\mathbf{1}$ presumably due to smaller crystalline domains. F urthermore, the polymerized bilayers were mechanically stable and the material remained even after treatments with organicsolvents and detergent solutions. Patterned polymeric bilayers could be created also on chitosan layers (data not shown). To

(51) The boundaries between the polymerized and nonpolymerized parts are unclear in Figure 8. This broadened interface is due to the stray light during the lithographic exposure process rather than propagation of the polymerized domains.

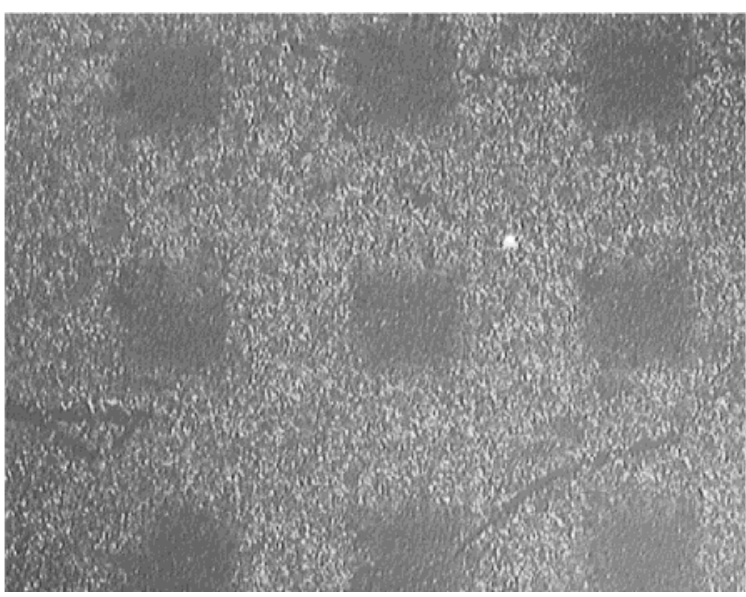

Figure 8. Differential interferencecontrast image of a $\mathbf{1}$ bilayer that was polymerized with a mask (UV irradiation, cross-linker, $200 \mathrm{~mJ}$ ). The polymerized bilayer area (grid) surrounds square corrals of monomeric $\mathbf{1}$ bilayers. The size of the corrals was 50 $\mu \mathrm{m}$.
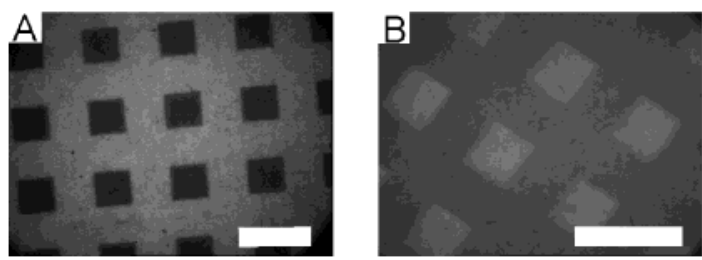

Figure 9. Fluorescence microscope images of patterned 2 bilayers on a glass. (A) The brightly fluorescent area (grid) was polymerized by UV irradiation (Pan-Ray, $60 \mathrm{~min}$ from $10 \mathrm{~cm}$ distance). In the square corrals $\mathbf{2}$ molecules were protected by the mask and remained monomeric. (B) Monomeric bilayers of $\mathbf{2}$ were removed with ethanol and lipid bilayers of egg-PC containing $1 \mathrm{~mol} \%$ NBD-PE were introduced into the same corrals by the vesicle fusion method. The square corrals are brightly fluorescent due to NBD-PE molecules. The scale bars correspond to $100 \mu \mathrm{m}$

understand the different behaviors of polymerized $\mathbf{1}$ and $\mathbf{2}$, wecould consider twofactors that are potentially related to the stability of polymerized bilayers. First, $\mathbf{2}$ has two diacetylene moieties in a singlemolecule. Thecross-linked network of polymeric chains formed within the bilayer is expected to makethe film mechanically robust. ${ }^{52}$ Second, bilayers of $\mathbf{1}$ apparently have a higher degree of crystallinity compared with $\mathbf{2}$, as suggested by its larger crystalline domains and efficient polymerization. This might result in theformati on of sharp domain boundaries and packing defects, leading toa lower mechanical stability of the membrane on the substrate.

Stability of polymerized bilayers depended on the duration of the UV light irradiation for photopolymerization, as well. Bilayers of $\mathbf{2}$ that were polymerized by relatively short irradiation were dissolved by ethanol or SDS solutions and mostly removed from the substrate surface. The polymeric bilayers became resistant toward solubilization, only if the UV irradiation time was sufficiently long. ${ }^{53}$ The UV-vis absorption band of the polymerized 2 bilayers at $470 \mathrm{~nm}$ grew rapidly upon UV light irradiation, indicating the rapid formation of conjugated polymeric backbones. However, the intensity of this spectral band was not directly correlated to the stability of polymerized bilayers. It is partially because

(52) Currently we do not have direct information on the degree of cross-link, since it is not accessible from the spectroscopic data.

(53) In the case of irradiation with a Pen-Ray lamp from a distance of $5 \mathrm{~cm}$, an exposure time of $10 \mathrm{~min}$ or longer was necessary to ensure the formation of stable polymeric bilayers. 
the effective conjugation length is limited by the local conformations of polymer backbones, and the absroption band does not represent longer pol ymer chains that might exist in the bilayers. More importantly, the absorption spectra do not give information concerning the degree of cross-linking between the polymer chains. Therefore, although we do not have information on the degree of cross-linking, our interpretation of the observed stability changes by the UV light irradiation time is that the bilayers have longer and more cross-linked polymeric backbones, if one photopol ymerizes them for longer time.

Since polymerized $\mathbf{1}$ bilayers were found to be mechanically unstable, further investigation of patterned bilayers was carried out only with the bilayers of polymerized 2. After the photopolymerization, unreacted monomers were removed from the bilayer by immersing the samples in ethanol. Toincorporate new li pid bilayers into the lipid-free domains, we have fused small unilamellar vesicles (extruded through a filter with pores of diameter ca. $50 \mathrm{~nm}$ ) of phosphatidylchol ine from egg yolk (egg-PC) doped with $1 \mathrm{~mol} \%$ of thefluorescently label ed lipid NBDPE. Observation by the fluorescence microscope revealed that the areas where the monomeric bilayers had been removed were refilled with egg-PC bilayers and became intensely fluorescent due to the incorporated NBD-PE (Figure9B). It should benoted that the polymerized bilayer domains (grid), which arefluorescent in Figure 9A, appear dark in Figure 9B due to the strong fluorescence from the NBD-PE containing bilayers in the corrals. The incorporated egg-PC/NBD-PE bilayers retained thelateral fluidity at room temperature, and the polymerized bilayers acted as a barrier that confined the fluid bilayers. This was proven by the following two sets of experiments. First, NBD-PE molecules in thefluid bilayers were bleached by illuminating an intense light on a selected area of the membrane (the illumi nation spot diameter was typically ca. $100 \mu \mathrm{m})$. If an egg-PC bilayer was located in a square corral surrounded by polymeric bilayers, bleaching of NBD-PE molecules within the corral resulted in complete loss of fluorescence from the particular corral and no recovery of fluorescence was observed (F i gure 10A before, and Figure 10B after bleaching). Illumination of the polydiacetylene bilayer with the same intensity and time did not result in a decrease of fluorescence intensity, showing the high photostability of the polydiacetylene matrix and the absence of NBD-PE in these regions. On the other hand, if the polymeric bilayers were created as parallel lines, the areas between them formed narrow channels of fluid lipid bilayers. In such a geometry, eggPC and NBD-PE molecules could diffuse through the channels. Therefore, photobleaching of NBD-PE resulted in temporal depression of fluorescence within the ilI umi nated spot and along the connected channel. Parts C and D of F igure 10 illustratefluorescencegradient created by the photobleaching and diffusion of NBD-PE molecules. In addition tothefluorescencemicrograph shown in Figure 10C, a schematic drawing is given in Figure 10D for clarity. Note the gradient of the brightness in Figure 10C (from top right through bottom left) that indicates the concentration gradient of NBD-PE molecules.

The second experiment to visualize the role of polymerized domains as a barrier was the diffusion of negatively charged NBD-PE in an applied electric field. This technique has been used previously to prove the confinement of lipids in defined geometries and to determine their lateral diffusion constants. ${ }^{54} \mathrm{~A}$ horizontal electric field

(54) Groves, J . T.; Boxer, S. G.; McConnell, H. M. Proc. Natl. Acad. Sci. U.S.A. 1997, 94, 13390-13395.
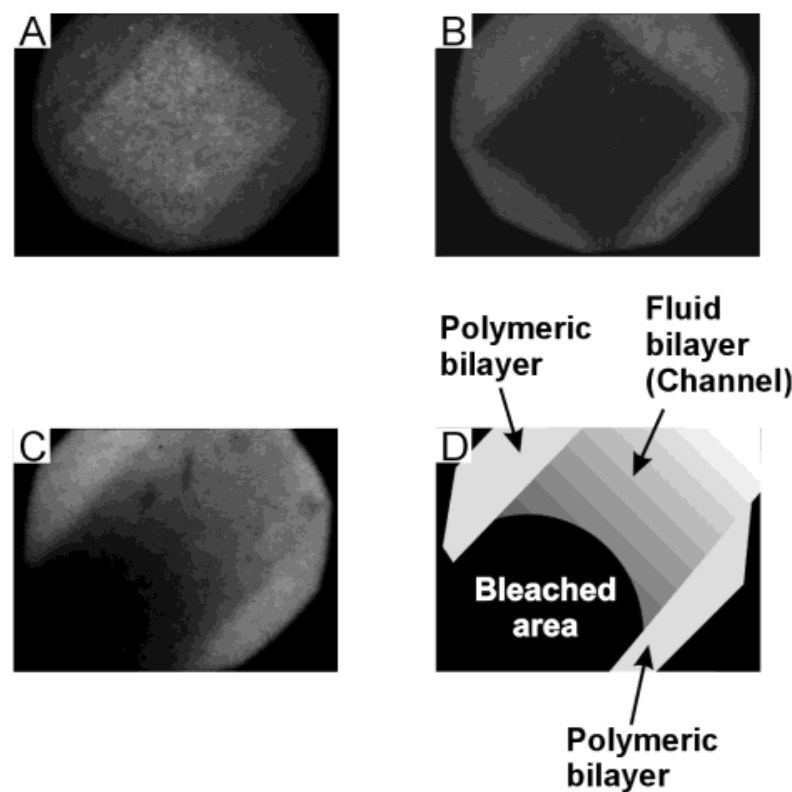

Figure 10. Fluorescence micrographs of egg-PC/NBD-PE bilayers incorporated intothewells between polymeric bilayers. A single corral and a channel (size $50 \mu \mathrm{m}$ ) were bleached by a strong illumination under the microscope objective. In the case of the corral, the fluorescence of NBD-PE was bleached compl etely (from (A) to (B)), wher eas the channel retained the fluorescence partially, because NBD-PE molecules were supplied continuously by diffusion (C). (The molecules at the left bottom side of the channel were bleached and new molecules were supplied from the right upper side (concentration gradient).) (D) Schematic drawing of the image in (C) that depicts the fluid bilayer channel and the concentration gradient of NBD-PE.

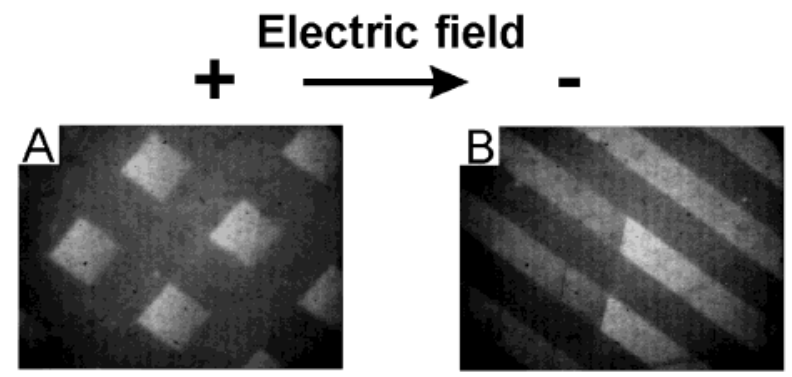

Figure 11. Fluorescencemicrographs of the patterned bilayers with incorporated egg-PC/NBD-PE bilayers in each corral under the influence of an electric field $(E)$ in the direction of the indicated arrow. The negatively charged NBD-PE molecules in the fluid bilayer membranes drifted and formed concentration gradients. The molecules were blocked by barriers of the polymerized bilayer (A) or by a line defect within the channels (B). The size of the corrals and channels (width) was $50 \mu \mathrm{m}$.

was applied by using a homemade el ectrophoresis cell. Under the applied electric field charged lipid molecules (in thecurrent caseNBD-PE) diffuse preferentially to one direction and build a concentration gradient (Figure 11). It can be seen in these pictures that NBD-PE molecules are accumulated at the sites where their diffusion is hindered. In Figure 11A, diffusion of NBE-PE was blocked at the left side of the corrals (the boundaries between the fluid and polymerized membrane domains). On the other hand, the diffusion of NBD-PE can be seen in Figure 11B along two channels that were partially blocked by a line defect across thechannels. Theseresults strongly indicate that the rigid polymeric bilayers of $\mathbf{2}$ and phosphatidylcholine bilayers are forming an integrated composite membrane system, where the fluid phosphatidylcholine 
bilayers are spatially confined by the micropatterned barriers of polymerized $\mathbf{2}$ bilayers.

\section{Conclusion}

Photopol ymerization of diacetylene amphiphiles $\mathbf{1}$ and 2 in SPB showed strong dependency on the underlying substrates. Most notably, a thin polymer layer covering the substrate surface could alter the polymerization process of the bilayers significantly. The observation that polymerization occurs also on polymeric materials, such as PEI and chitosan, is an important step toward the construction of polymer-supported patterned lipid membane systems envisaged in Figure 1 . The reconstitution of fluid bilayer membranes on polymeric support layers represents an important technical challenge to be addressed in the future studies. ${ }^{55}$ The comparison between two types of diacetylene amphiphiles, the single chain alkyl phosphate $\mathbf{1}$ and the double chain phospholipid 2, yielded insight intotheimportance of the typeand nature of the polymerization process for the fabrication of twodimensional micropatterned structures. The singlechain diacetylene amphiphile $\mathbf{1}$ polymerized much more efficiently than the double chain counterpart, owing to its favorablemolecular packing within thebilayer. However, at the same time it developed sharp domain structures with defects and made the polymerized film mechanically less stable. The double chain diacetylene phospholipid 2, on the other hand, had a less faborable packing of molecules for the polymerization. It resulted in a slower polymerization and a shorter conjugation length. Nevertheless, the formed polymeric bilayers showed a much

(55) Adsorption of SPB on PEI and chitosan polymer layers has been reported in previous studies (Wong, J . Y .; Majewski, J .; Seitz, M .; Park, C. K.; Israelachvili, J .; Smith, G. S. Biophys. J . 1999, 77, 1445-1457. Baumgart, T. Ph.D.Thesis, J ohannes Gutenberg Universität, Mainz, 2001.) In the current study, however, we have not investigated the incorporation of fluid egg-PC bilayers into the micropatterned bilayers on polymeric supports. higher stability. This is presumably due to the fact that each molecule has two polymerizable groups and the formed polymeric bilayer should contain some degree of cross-linking. Furthermore, the packing difficulties of the phospholipid seem to prevent the formation of sharp domain boundaries. These observations lead us to the realization that an amorphous, highly cross-linked network should be built within the polymerized bilayer, so that it can stabilize the whole membrane structure most effectively. ${ }^{56}$ Our current study has demonstrated that patterned polymeric bilayers of $\mathbf{2}$ can act as a template to incorporate lipid bilayer membranes. The micropatterning strategy based on lithgraphicphotopolymerization of lipid bilayers should provide new possibilities to construct complex and versatile biomimetic membrane systems by enhancing mechanical stability, creating spatial definition, and thus enabling the design of additional functions.

Acknowledgment. Wethank Professor H. Ringsdorf (J ohannes Gutenberg Universität, Mainz) for the kind gift of polymerizable monoalkyl phosphate 1, Mr. B. Zimmer for his support in the fluorescence spectroscopy measurements, and Dr. H. Schönherr (University of Twente, The N etherlands) for critical reading and hel pful comments on the manuscript. The work was supported by theMinisterium für Bildung, Wissenschaft, F orschung und Technologie (BMBF) (Project No. 0310852), the Schloesmann Foundation Fellowship (K.M.), and Graduierten Kolleg Stipendium (T.B.).

\section{LA0157420}

(56) Considering the fact that diacetylene lipids are rather brittle crystalline materials, one could possibly achieve a better mechanical stabilization by empl oying less rigid polymerizablelipids. F or example, some sorbyl-group-based lipid bilayers were recently demonstrated to polymerize on solid substrates. (Ross, E. E.; Bondurant, B.; Spratt, T.; Conboy, J . C.; O'Brien, D. F.; Saavedra, S. S. Langmuir 2001, 17, 23052307.) This could be regarded as one of the attractive candidates for the construction of patterned polymeric bilayers. 